\title{
Epigenetic Changes Associated With Interleukin-10
}

\author{
Zhonghua Zheng, Gang Huang, Tong Gao, Tianyi Huang, Mengsha Zou, Yuhao Zou and \\ Shiwei Duan*
}

Medical Genetics Center, School of Medicine, Ningbo University, Ningbo, China

$\mathrm{IL}-10$ is a regulator of inflammation and immunosuppression. $\mathrm{IL}-10$ regulates a variety of immune cells to limit and stop the inflammatory response, and thus plays an important role in autoimmune diseases, inflammatory diseases and cancer. IL-10 is closely related to epigenetic modification, in which changes in DNA methylation of IL-10 gene can affect mRNA and protein levels of IL-10. In addition, changes in histone modifications, especially histone acetylation, can also lead to abnormal expression of IL-10 mRNA. At the same time, a handful of $\mathrm{IL}-10$ related microRNAs (miRNAs) are found to be aberrantly expressed in multiple diseases. Besides, long non-coding RNA (IncRNA) growth arrest specific transcript 5 (GAS5) also inhibits IL-10 expression. Here, we reviewed the epigenetic changes related to $\mathrm{IL}-10$ in various diseases, as well as the regulation of IL-10 gene expression in various diseases by epigenetic modifications such

OPEN ACCESS

Edited by:

Masato Kubo,

Tokyo University of Science, Japan

Reviewed by:

Luciana D'Apice,

National Research Council (CNR), Italy

Yoshihiro Baba,

Kyushu University, Japan

${ }^{*}$ Correspondence:

Shiwei Duan

duanshiwei@nbu.edu.cn

Specialty section:

This article was submitted to

Cytokines and Soluble Mediators in

Immunity,

a section of the journal

Frontiers in Immunology

Received: 01 October 2019

Accepted: 06 May 2020

Published: 04 June 2020

Citation:

Zheng Z, Huang G, Gao T, Huang T,

Zou M, Zou Y and Duan S (2020)

Epigenetic Changes Associated With

Interleukin-10.

Front. Immunol. 11:1105.

doi: 10.3389/fimmu.2020.01105 as DNA methylation, histone modification, miRNA, and IncRNA.

Keywords: DNA methylation, epigenetics, interleukin-10, immune inflammatory disease, histone modification, microRNA, IncRNA

\section{INTRODUCTION}

As the primary member of the IL-10 cytokine family (1), IL-10 plays an important role in the regulation of the differentiation and proliferation of a variety of immune cells, such as $\mathrm{T}$ cells, $\mathrm{B}$ cells, natural killer cells, antigen presenting cells, mast cells, and granulocytes (2). The transcription mechanism of IL-10 is also very complicated. Foxp3, Nfil3, Ets1, and other transcription factors can modify the chromatin state of IL-10 by recruiting various effector proteins (3). The major biological function of IL-10 is to limit and stop the inflammatory response, which is pivotal in autoimmune diseases, inflammatory diseases and cancer $(4,5)$.

Epigenetics refers to modifications in the genome that do not alter the DNA sequence, including DNA methylation, histone modifications, non-coding RNA, etc. (6). Epigenetic modifications can respond to environmental stimuli by activating or inhibiting gene transcription (7). Recent studies have shown that epigenetic regulation of IL-10 expression can influence the progression of diseases such as Behçet's's disease and atherosclerosis $(8,9)$.

\section{IL-10 STRUCTURE}

IL-10 is a regulator of inflammation and immunosuppression (10). Human IL-10 gene is 4,893 bp in length and includes 5 exons and 4 introns, which are located at 1q32.1, encoding genes on the minus strand. The human functional IL-10 protein is a dimer of 160 amino acids after removal of the 18 amino acid signal peptide $(11,12)$. Each strand of mouse IL-10 (mIL-10) consists of 157 amino acids and its amino acid sequence is $\sim 75 \%$ identical to human IL-10 (13). There are some important single nucleotide polymorphisms in the IL-10, including -819T/C (rs1800871), 
-592A/C (rs1800872), and -1082G/A (rs1800896), etc. (14). These genetic variants can regulate IL-10 expression levels, thereby affecting the progression and severity of IL-10-related diseases $(15,16)$.

\section{DNA METHYLATION MODIFICATION ASSOCIATED WITH IL-10}

The IL-10 protein is ubiquitously expressed in various tissues of mice, and the methylation level of the IL-10 gene is not high and specific in each tissue (17). As shown in Figure 1, DNA methylation regulates IL-10 expression in different cells. Larsson et al. have shown that the methylation status of the human IL-10 promoter is closely related to the transcriptional activity of IL-10
(18). Specially, in human peripheral blood mononuclear cells, the hypomethylation of the IL-10 promoter corresponds to higher IL-10 expression; in the epithelial cells, the IL-10 promoter shows hypermethylation to silence IL-10 expression (18). Hedrich et al. have shown that the IL-10 gene intron has an enhancer, and the decrease in DNA methylation of the enhancer in $\mathrm{T}$ cells of systemic lupus erythematosus (SLE) patients can increase the recruitment of STAT transcription factors and promote IL10 expression (19). Besides, Yu et al. have found that arginine can induce hypomethylation of IL-10 promoter DNA, thereby regulating IL-10 production in neonatal Treg cells (20). The methylation status of the IL-10 locus promoter in Th1 cells can be reversibly regulated, which may be used to rapidly regulate the transcription level of IL-10 gene (21). Lorente-Sorolla et al. found

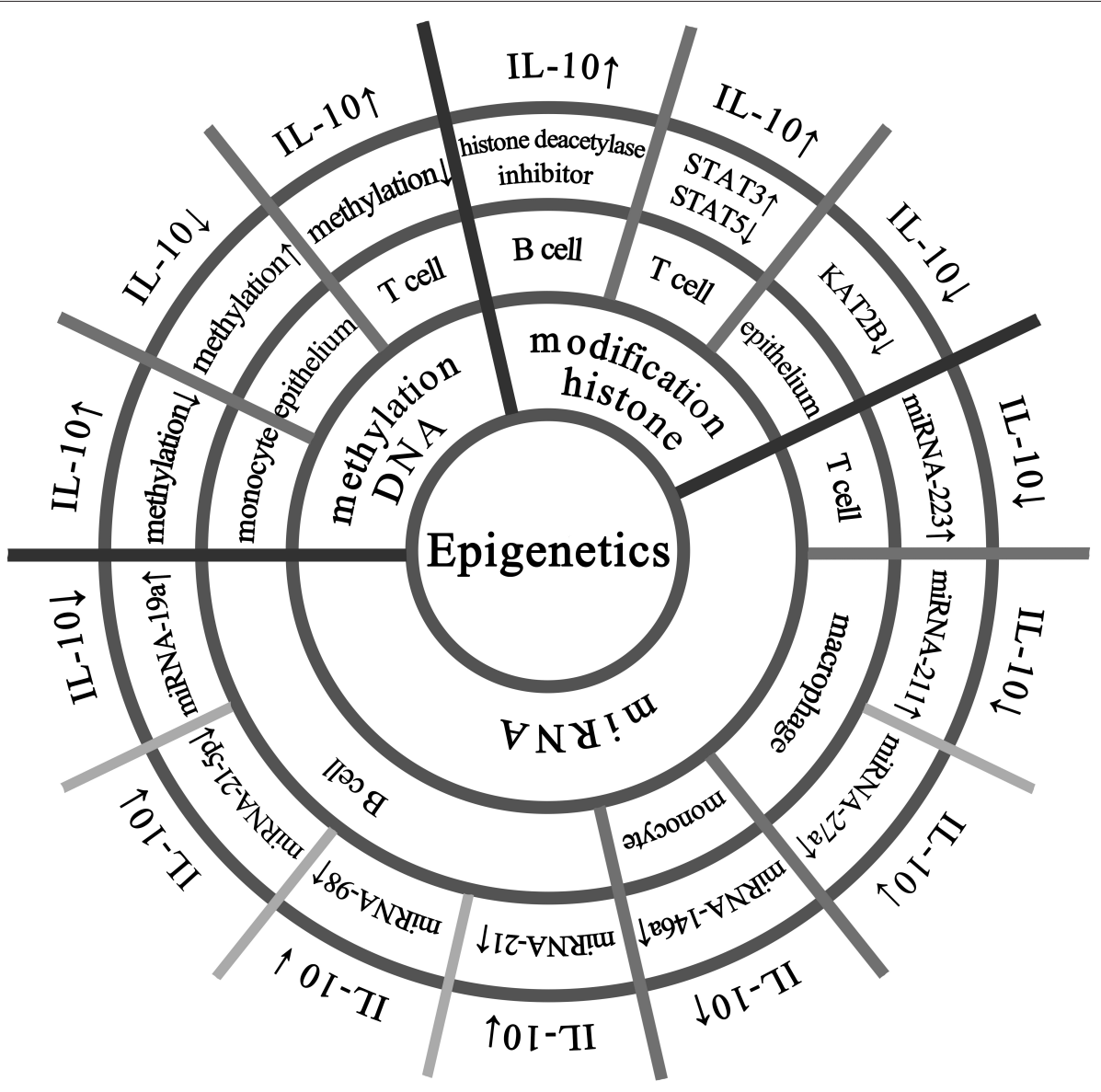

FIGURE 1 | Epigenetic modifications that regulate IL-10 expression in multiple cells. The IL-10 promoter has transcriptional activity and hypomethylation in human peripheral blood mononuclear cells, while the IL-10 promoter is silenced in epithelial cells due to hypermethylation. There is an enhancer in the IL-10 gene intron in T cells, and its hypomethylation promotes IL-10 expression. B cells treated with histone deacetylase inhibitor and 5-aza can increase the expression of IL-10. In T cells, increased activation of STAT3 can lead to enhanced recruitment of regulatory regions and competitive replacement of STAT5, promoting IL-10 expression. In normal colonic epithelial cell line (NCM460), laccase acid inhibits KAT2B and reduces the transcriptional activity of KAT2B and H4K5ac on the IL-10 promoter, thereby significantly down-regulating the expression of IL-10. Overexpression of miRNA-146a in peripheral blood mononuclear cells increases IL-10 expression. Up-regulation of miRNA-19a in B cells reduces IL-10 expression, and the same up-regulation of miRNA-98 can also suppress IL-10 expression, and insufficient expression of miRNA-21-5p is one of the reasons for the decrease in IL-10 expression in B cells. Up-regulation of miRNA-21 inhibits the differentiation of IL-10 + Breg cells and promotes the expression of miRNA-223 in autoimmune T cells, resulting in reduced IL-10 production. In macrophages, the relative expression of miRNA-211 is abnormally upregulated, accompanied by a decrease in secreted IL-10. miRNA-27a can enhance the antibacterial activity of macrophages and inhibit the expression of IL-10. 
that increased IL-10 levels were associated with changes in IL-10 methylation in circulating monocytes in patients with sepsis (22). At present, DNA methylation modification has been recognized as a key regulatory mechanism for IL-10 gene expression (8).

As shown in Table 1, IL-10 DNA methylation levels are associated with many diseases. As the severity of liver failure increases, the level of IL-10 in serum of patients with hepatitis increases, and the decrease in methylation of IL-10 promoter plays an important role in IL-10 gene activation (23). IL-10 intron methylation levels were higher in asthmatic patients than in non-asthmatic patients, and changes in IL10 different methylation regions were associated with the exposure risks of $\mathrm{CO}, \mathrm{NO}_{2}$, and PM2.5 (24). In addition, the IL-10 gene is hypermethylated in periodontal tissues (27). Hypermethylation of CpG islands in the IL-10 promoter region may be involved in the development of rheumatoid arthritis (RA) (28). In addition, IL-10 gene methylation levels were decreased in SLE CD4+ T cells compared with healthy controls, and negatively correlated with IL-10 mRNA expression (30). The hypomethylated IL-10 gene is associated with higher SLE disease activity, and serum IL-10 levels are also reduced in RA patients. Therefore, IL-10 hypomethylation may provide a potential epigenetic marker for clinical prediction of autoimmune diseases (29). A study found that increased FOXP3-TSDR methylation levels were significantly associated with the severity of atherosclerosis and the changes in IL-10 concentration (31).

IL-10 promoters tend to be hypomethylated in different cancer types (32). Lima et al. showed that there were $37 \mathrm{CpG}$ sites with methylation differences between esophageal squamous cell carcinoma (ESCC) and adjacent non-tumor tissues, and these sites were associated with IL-10-related pathways including antiinflammatory signaling pathways, and cellular communication pathways, etc. (33). Alipour et al. collected blood samples from 51 Behçet's disease patients and 63 healthy controls, evaluated promoter methylation levels by MeDIP-qPCR, and subsequently assessed IL-10 expression by Real-time PCR (8). And their study found that the expression level of IL-10 gene was significantly decreased in blood samples from patients with Behçet's disease, mainly due to the high methylation of the IL-10 promoter region resulting in decreased mRNA expression levels (8). In addition, IL-10 promoter hypomethylation and IL-10 overexpression were observed in breast cancer tumor tissues compared to normal breast tissue and benign breast tissue (25). Drennan et al. showed that IL-10 gene hypomethylation is a prominent feature of chronic lymphocytic leukemia (26). At the same time, Tonon et al. found that IL-10 demethylation corresponds to IL-10 expression in mouse and human B cellassociated cancers (34). Epigenetic changes, such as abnormal DNA methylation of IL-10, are also closely associated with the development and progression of colorectal cancer (35). The main thrust of our review is to introduce the epigenetics of IL-10, so we have not introduced many studies on IL10 polymorphism.

\section{IL-10 DNA METHYLATION LEVELS MAY BE AN IMPORTANT FEATURE OF THE DISEASE}

The level of IL-10 DNA methylation is affected by a variety of factors, which presents different levels of modification in different diseases and different tissues. The severity of the disease affects the methylation level of IL-10. Hepatitis patients with increased liver failure will lead to IL-10 promoter methylation and IL10 gene inactivation. In addition, IL-10 methylation increased significantly during short and long-term exposure to high levels of $\mathrm{CO}, \mathrm{NO}_{2}$, and PM2.5. In cancer, the IL-10 promoter is often hypomethylated. In summary, the level of IL-10 methylation modification may become a marker for diagnosis and prognosis of disease.

\section{HISTONE MODIFICATIONS ASSOCIATED WITH IL-10}

Epigenetic modifications of DNA-binding proteins, such as histone acetylation, are also important factors influencing gene expression (36). A handful of studies have found that histone modifications can affect IL-10 level (Figure 2). Treatment

TABLE 1 | Relationship between IL-10 DNA methylation and various diseases.

\begin{tabular}{|c|c|c|c|c|}
\hline Disease & Samples (n) & Method & Methylation level & References \\
\hline ACLF & ACLF (25), CHB (25), Healthy controls (10) & MSP & Hypermethylation & $(23)$ \\
\hline Asthma & Students of Fresno Unified School (188) & Bisulfite pyrosequencing & Hypermethylation & $(24)$ \\
\hline BD & Peripheral blood of patients (51), Healthy controls (63) & MeDIP-qPCR & Hypermethylation & (8) \\
\hline Breast cancer & Breast cancer tissues (72), Benign tissues (31), Normal tissues (30) & Bisulfite sequencing & Hypomethylation & $(25)$ \\
\hline CLL & CLL (27, CSU cohort), CLL (36, DKFZ cohort) & BSP & Hypomethylation & $(26)$ \\
\hline Periodontal disease & Gingival tissue (34) & MSP & Hypermethylation & $(27)$ \\
\hline RA & RA patient (34), Healthy controls (30) & MSP & Hypermethylation & $(28)$ \\
\hline SLE & SLE (66), RA (12), Healthy controls (102) & Whole genome methylation array & Hypomethylation & $(29)$ \\
\hline SLE & SLE (30), Healthy controls (26) & Bisulfite Sequencing & Hypomethylation & (30) \\
\hline
\end{tabular}

ACLF, acute on chronic liver failure; BD, Behçet's disease; CLL, chronic lymphocytic leukemia; CSU, Cancer Sciences Unit in Southampton; DKFZ, German Cancer Research; RA, rheumatoid arthritis; SLE, systemic lupus erythematosus. 


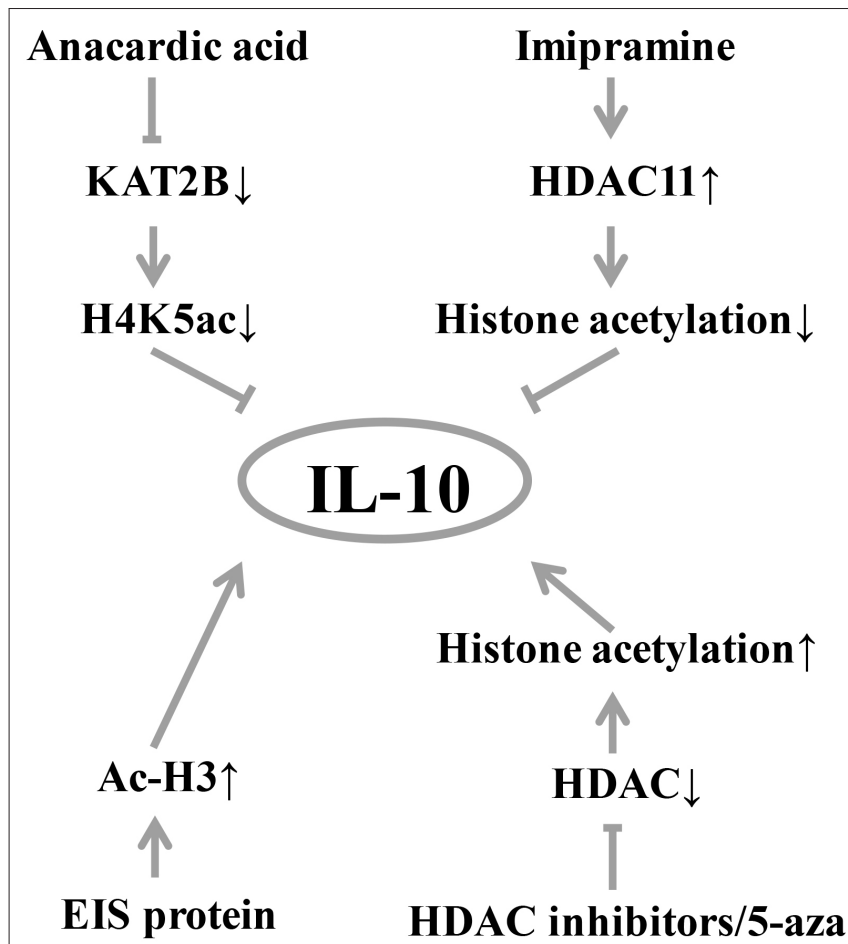

FIGURE 2 | Diagram of IL-10 and histone modification. Histone acetylation induces the production of analgesic factors such as IL-10. EIS protein can significantly increase the level of acetylation of histone $\mathrm{H} 3(\mathrm{Ac}-\mathrm{H} 3)$, thereby increasing the expression of IL-10. Histone acetylase inhibitors and 5-aza treatment increased IL-10 mRNA expression. Inhibition of histone deacetylase by histone deacetylase inhibitors, histone acetylation induces spinal cord production of analgesic factors including IL-10. Conversely, the inhibition of anacardic acid down-regulated KAT2B, thereby reducing the occupancy of KAT2B and H4K5ac by the IL-10 promoter, resulting in down-regulation of $\mathrm{IL}-10$ expression by transcriptional silencing. Similarly, imipramine upregulates histone deacetylase 11, which inhibits the acetylation of the IL-10 promoter resulting in a decrease in IL-10 production. KAT2B, lysine acetyltransferase 2B; H4K5ac, histone $\mathrm{H} 4$ lysine 5 acetylation; HDAC, histone deacetylase; Ac-H3, acetylation level of histone H3; EIS, enhanced intracellular survival.

of $\mathrm{B}$ cells with histone deacetylase inhibitor and 5-aza-2'deoxycytidine (5-aza) increases IL-10 mRNA transcription (18). In a mouse experiment, histone acetylation induces spinal cord production of analgesic factors including IL-10 $(37,38)$. In T cells from SLE patients, increased expression of STAT3 promotes its binding to the regulatory region of IL-10 and competitively replaces the binding of STAT5 to the regulatory region of IL-10, which subsequently promotes IL-10 gene expression (19). In the normal colonic epithelial cell line (NCM460) (Figure 1), anacardic acid down-regulates the KAT2B gene, reducing the transcriptional activity of KAT2B and $\mathrm{H} 4 \mathrm{~K} 5 \mathrm{ac}$ on the IL-10 promoter, thereby significantly downregulating IL-10 expression (39). In addition, imipramine upregulates histone deacetylase 11, which inhibits the binding of the acetylated histone of the IL-10 gene promoter and reduces IL-10 expression (40). Duan et al. found that enhanced intracellular survival (EIS) protein significantly increased the acetylation level of histone $\mathrm{H} 3$ (Ac-H3), which in turn increased the binding of Ac-H3 to the SP1 and STAT3 regions of the human
IL-10 gene promoter, thereby promoting the expression of IL$10(41)$.

\section{IMPORTANT ROLE FOR HISTONE MODIFICATION OF IL-10}

As shown in Figure 2, treatment of B cells with histone deacetylase inhibitor and 5-aza can increase the expression of IL-10 mRNA, and the inhibition of KAT2B by ursolic acid can significantly down-regulate the expression of IL10. In addition, the up-regulation of imipramine can reduce IL-10 production.

\section{IL-10-ASSOCIATED MIRNA}

The miRNAs are a class of non-coding RNA molecules ranging in length from 18 to 24 nucleotides (42). In peripheral blood mononuclear cells, IL-10 expression is inversely correlated with the level of miRNA-98-5p (43). Overexpression of miRNA-146a increased IL-10 expression in peripheral blood mononuclear cells [Figure 1; (44)]. In breast cancer cells, down-regulation of miRNA-141 inhibits IL-10 and leads to up-regulation of COX-2, PGE-2, and TNF- $\alpha$ expression (45). Increased IL10 release after hip fracture can lead to significant systemic inflammatory response and acute lung injury (ALI) after hip fracture, and increased IL-10 release is significantly associated with up-regulation of miR-146a and down-regulation of miRNA150 (46). Besides, miRNA-10a is found to be positively correlated with serum IL-10 concentration and plays an important role in atherosclerotic inflammation (47). miRNA-155 has been shown to play a role in immune activation and inflammatory responses and is inhibited by IL-10 $(48,49)$. Similarly, IL-10 has been found to inhibit TLR4-induced miRNA-155 expression (50). The antagonism between IL-10 and miRNA-155 is necessary to balance host defense and immune activation in vivo, and this balance is particularly important in inhibiting Lyme myocarditis (51). Besides, TNF- $\alpha$, IFN- $\gamma$, and IL-4 inhibit IL-10 expression in B cells by up-regulating miRNA-19a expression (52, 53). Liu et al. found that miRNA-19a levels were higher in B cells of sensitized mice in inducing intestinal allergy-related inflammation, thereby inhibiting IL-10 expression in B cells (54). The miRNA-210 expression was significantly elevated in placenta of preeclampsia (PE) patients, while IL-10 levels were significantly lower than normal pregnant women (55). Luo et al. found that the expression of IL-10 was positively correlated with the expression of miRNA-21-5p in B cells of patients with allergic purpura (HSP) (56). In BALF-derived macrophages from ARDS rats, the relative expression of rno-miRNA-211 was up-regulated, accompanied by a decrease in secretory IL10 (57). Lu et al. have shown that increased expression of miRNA-223 in T cells of RA patients leads to a decrease in insulin-like growth factor-1 mediated IL-10 production, leading to an imbalance between pro-inflammatory cytokines and antiinflammatory cytokines (58). Sun et al. reported that rnomiRNA-30b-5p is down-regulated in the spleen, lymph nodes, and eye tissues of experimental autoimmune uveitis rats, while 
down-regulation of rno-miRNA-30b-5p may be regulated by IL10 and TLR 4 levels affect the proportion of IL-10 positive cells in the cell population, thereby inhibiting the development of uveitis. rno-miRNA-30b-5p mimics can reduce the expression of IL-10 and TLR4 genes and proteins, thereby affecting the pathogenesis of uveitis. Therefore, rno-miRNA-30b-5p may be a new therapeutic target for uveitis (59). In addition, studies have shown that miRNA-410 is a key regulator of the pathogenesis of SLE, and it regulates IL-10 expression by targeting STAT3 (60). Li et al. showed that down-regulation of miRNA-98 was accompanied by up-regulation of IL-10 in tumor-associated macrophages of hepatocellular carcinoma (HCC), suggesting that IL-10 is a direct target of miRNA-98 (61). Li et al. showed that administration of liposomes carrying miRNA-98 could effectively reduce the frequency of $\mathrm{B} 10$ cells in tumor-bearing mice and inhibit experimental tumor growth, demonstrating that IL-10-producing B cells play an important role in tumor tolerance effect, while miRNA-98 up-regulation can suppress the expression of IL-10 in B cells, which helps suppress the body's tolerance to lung cancer (62). At the same time, miRNA-98 also plays an important role in myocarditis by inhibiting the expression of IL-10 in cardiac B cells (63). The miRNA-98 is involved in the transcriptional regulation of interleukin-10 in peripheral blood B cells of patients with airway allergy (64). Furthermore, inhibition of miRNA-98 reverses the ability of IL10 expression in B cells (9). In addition, Rouas et al. showed that miR-27b-3p and miR-330-3p may be involved in posttranscriptional control of the immunosuppressive cytokines IL10 and TGF- $\beta$ (65). The miRNA-21 inhibits the differentiation of IL-10+ Breg cells and promotes autoimmunity by targeting the 3 ' untranslated region of IL-10 mRNA (66). Other studies have shown that histone acetyltransferase p300 is involved in IL4/miRNA-98/IL-10 axis in peripheral blood B cells of patients with airway hypersensitivity. Therefore, p300 inhibitors have therapeutic potential in the treatment of allergic diseases (64). Xiong et al. found that IL-10 negatively regulates miRNA7025-5p, which down-regulates osteoblast differentiation. In vivo studies have shown that pre-injection of IL-10 results in increased bone formation, while injection of miRNA-7025-5p delays fracture healing. Therefore, IL-10 represents a promising therapeutic strategy to promote fracture healing (67). Li et al. found that miRNA-4492 was down-regulated in nasal polyps (NPs), while IL-10 was up-regulated in NPs, and the two were inversely related. This suggests that miRNA-4492/IL-10 axis

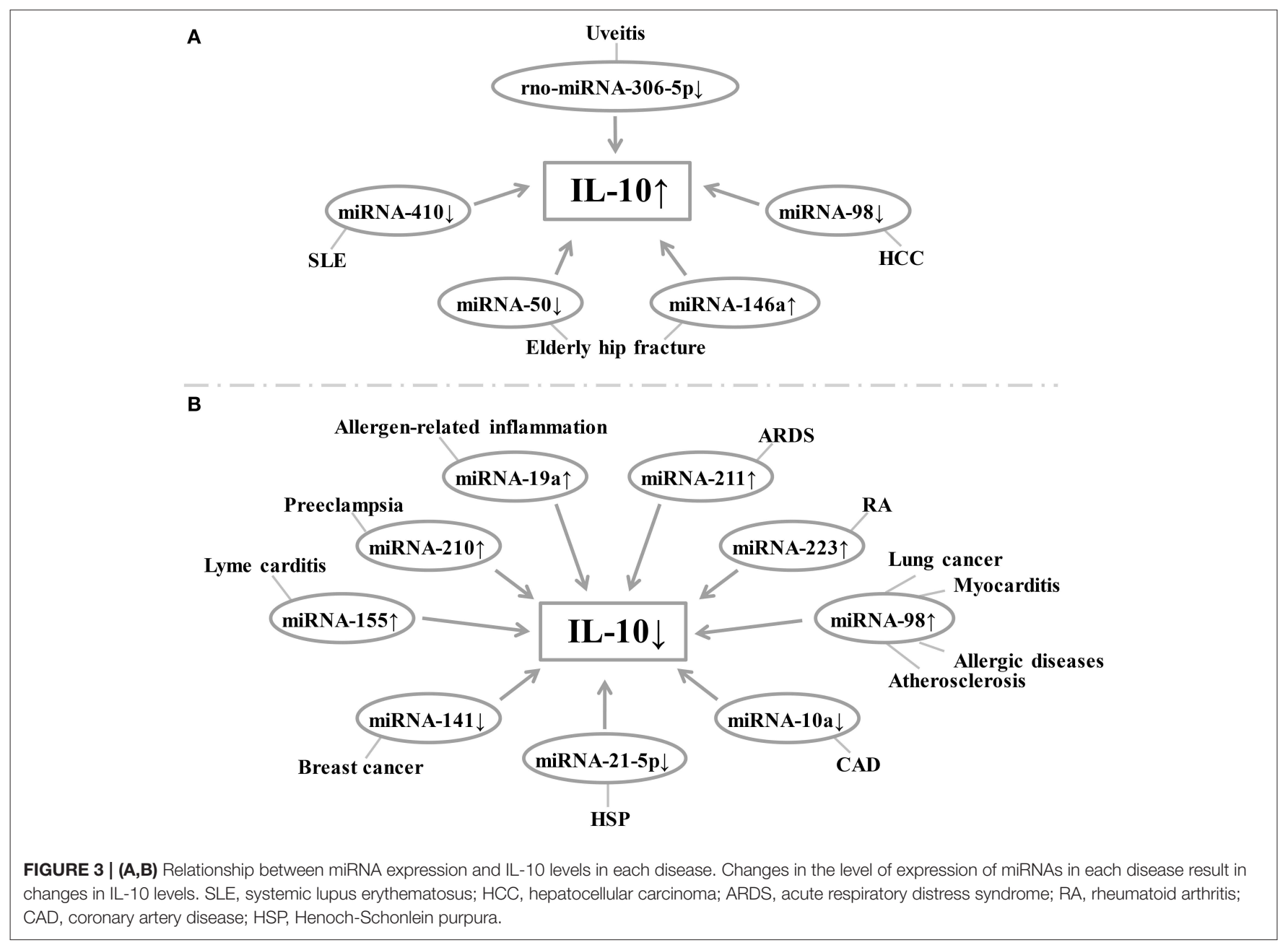


involvement in the Jak/STAT signaling pathway may be a key mechanism for chronic sinusitis with NPs (68). Hatab et al. found that in patients with advanced liver cancer, 3 consecutive months of curcumin, piperine, and taurine combined treatment could reduce circulating levels of IL-10 and miRNA-21, and patients with high baseline levels of IL-10 and miRNA-21 had a worse overall survival rate. Therefore, changes in serum IL10 and miRNA-21 expression may be prognostic biomarkers in the treatment of HCC (69). Finally, miRNA-27a enhances the antibacterial activity of macrophages and inhibits the expression of IL-10, thereby regulating the innate immune response (70). The relationship between miRNA and IL-10 levels in various diseases is summarized in Figure 3 and Table 2.

\section{REGULATORY miRNAs TARGETING IL-10 MAY PROVIDE NEW THERAPEUTIC TARGETS FOR MANY DISEASES}

IL-10 is a regulator of inflammation and immunosuppression. It regulates a variety of immune cells to limit and stop the inflammatory response, so it plays an important role in autoimmune diseases, inflammatory diseases, and cancer. Therefore, the regulatory effect of miRNA on IL-10 can be applied to many diseases that show changes in IL-10 levels, for the treatment or prognosis of diseases. Serum miRNA146a and miRNA-150 can be used as biomarkers for diagnosis and prediction of ALI (46), and rno-miRNA-30b-5p is a new therapeutic target for uveitis (59). It is worth noting that miRNA98 can be involved in the treatment of various diseases, such as lung cancer, liver cancer and other tumors, as well as myocarditis and allergic diseases $(61,62,64,71)$. In summary, regulatory miRNAs targeting IL-10 provide new therapeutic targets for many diseases, and also provide ideas for the development of new drugs for immune and inflammatory diseases in the future.

\section{LncRNA ASSOCIATED WITH IL-10}

LncRNA is defined as a long RNA transcript of more than 200 nucleotides that cannot be translated into a protein (72). Increasing amount of studies have shown that lncRNA plays a key role in the occurrence and development of cancer. Tang et al. found lncRNA actin filament-associated protein 1 antisense

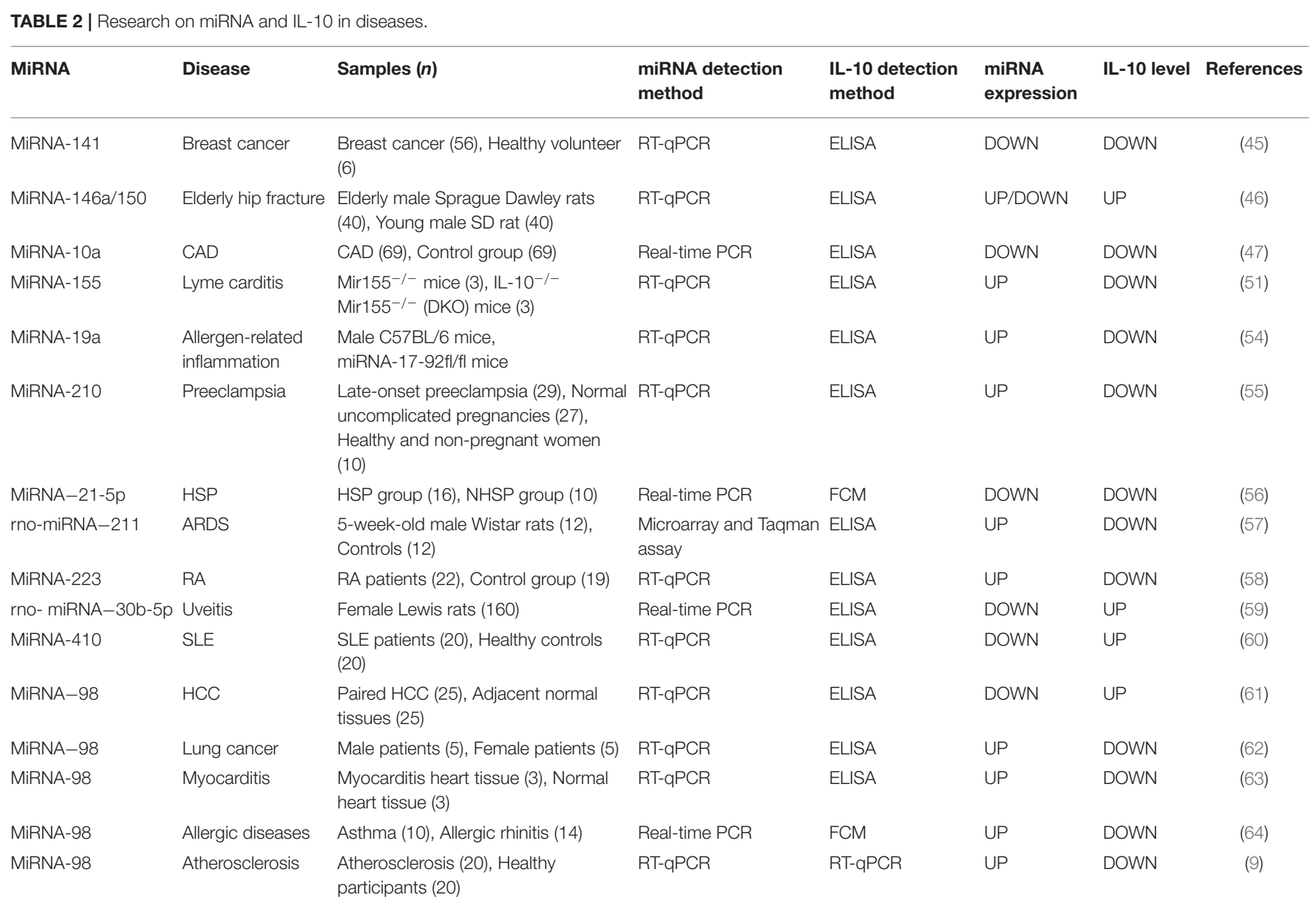

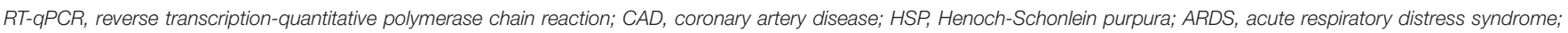
$R A$, rheumatoid arthritis; SLE, systemic lupus erythematosus; HCC, hepatocellular carcinoma; FCM, flow cytometry. 
RNA 1 (AFAP1-AS1) was highly expressed in non-small cell lung cancer (NSCLC), accompanied by increased expression levels of IL-10 and interferon (IFN)- $\gamma$ (73). Pei et al. found that IncRNA SNHG1 was increased in CD4+ TIL cells of breast cancer patients, while siRNA-SNHG1 can reduce the expression of Foxp3 and IL-10 (74). Ye et al. showed that lncRNA cox-2 siRNA can increase the expression levels of IL10, Arg-1, and Fizz-1 in M2 macrophages (75). Zhou WY reported that the silencing of lnc-LINC00473 can reduce the expression of IL-10 in B-cell (76). In addition, Ma P. et al. found that overexpression of lnc-MALAT1 can increase IL-10 expression, thereby inhibiting neuronal apoptosis and promoting axon growth (77). Shi et al. found that increased expression of lncRNA-MALAT1 can decrease IL-10 expression, thereby upregulating neuronal apoptosis and aggravating brain injury in rats with cerebral infarction (78). Li et al. found that lncRNA growth arrest specific transcript 5 (GAS5) was down-regulated in colorectal cancer (CRC), and subsequent functional experiments revealed that knockout of GAS5 promoted CRC cell proliferation and colony formation, while knockdown of GAS5 increases IL10 expression and reduced CRC cell proliferation and colony formation (79).

\section{IncRNA AND IL-10: A NEW RESEARCH DIRECTION}

Although lncRNA has become a research hotspot, we find that there is very little related research on lncRNA and IL-10. Therefore, there may be new discoveries related to lncRNA in the future research direction of IL-10.

\section{SUMMARY}

IL-10 is a highly potent cytokine produced by a variety of cells. It regulates cell growth and differentiation, participates in inflammatory reactions, and immune responses, and is currently recognized as inflammation and immunosuppressive factor. It plays an important role in autoimmune diseases and inflammatory diseases. Therefore, it is also an important means of treating related diseases. Almost all lymphocytes in the body can synthesize IL-10, including mononuclear macrophages, DCs, B cells, etc. Increased IL-10 expression can cause immunosuppression and SLE, RA, asthma, gastric cancer,

\section{REFERENCES}

1. Ouyang W, Rutz S, Crellin N, K, Valdez PA, Hymowitz SG. Regulation and functions of the IL-10 family of cytokines in inflammation and disease. Annu Rev Immunol. (2011) 29:71109. doi: 10.1146/annurev-immunol-031210-101312

2. Asadullah K, Sterry W, Volk HD. Interleukin-10 therapy-review of a new approach. Pharmacol Rev. (2003) 55:241-69. doi: 10.1124/pr. 55.2 .4

3. Zhang $\mathrm{H}$, Kuchroo $\mathrm{V}$. Epigenetic and transcriptional mechanisms for the regulation of IL-10. Semin Immunol. (2019) 44:101324. doi: 10.1016/j.smim.2019.101324 and other diseases. Also, IL-10 plays a decisive role in the development of immune paralysis, the development of temporary immunodeficiency after trauma, major surgery, burns, shock, and high-risk bacterial/fungal infections.

There are many studies on the epigenetics of IL-10. In different diseases, the DNA methylation modification of IL-10 gene locus can affect the occurrence and development of the disease. In addition, miRNA-98 is an important miRNA, which is associated with IL-10 in various diseases, such as HCC, Lung cancer, Myocarditis, etc. $(61,62,71)$. These studies have shown that the epigenetic mechanism of IL-10 help us to have a deeper understanding of the occurrence and progression of the disease. However, the current IL-10 related epigenetics research has not yet involved the diagnosis and clinical treatment of diseases. A recent review (3) describes the epigenetic mechanism controlling IL-10 expression, with an emphasis on some transcription factors in the construction of chromatin landscape in IL-10 induced $\mathrm{T}$ helper cell (3). Here, we present the epigenetic mechanisms controlling IL-10 expression in various diseases, and we also review the regulation of IL-10 gene expression in terms of DNA methylation, histone modification, miRNA, and lncRNA.

Epigenetics plays an important role in the regulation of IL10. Changes in DNA methylation levels, histone acetylation or deacetylation affect IL-10 expression. At the same time, miRNA plays a regulatory role in the late IL-10 transcription, and the mechanism of circRNA and IL-10 related to immune inflammation is still unclear, and it is still a blank, providing ideas and directions for future research. The mechanisms of IL10 production and regulation are very complicated, and we need to continue to research and discover in the future.

\section{AUTHOR CONTRIBUTIONS}

$\mathrm{SD}$ and $\mathrm{ZZ}$ contributed to the conception, design and final approval of the submitted version, and writing the paper. $\mathrm{GH}$ and TG contributed to interpretation of data. TH, MZ, and YZ contributed to the completion of figures and tables. All authors have read and approved the final manuscript.

\section{FUNDING}

This research was supported by the grants from K. C. Wong Magna Fund in Ningbo University. 
8. Alipour S, Nouri M, Khabbazi A, Samadi N, Babaloo Z, Abolhasani S, et al. Hypermethylation of IL-10 gene is responsible for its low mRNA expression in Behcet's disease. J Cell Biochem. (2018). 119:6614-22. doi: 10.1002/jcb. 26809

9. Huo Y, Chu Y, Guo L, Liu L, Xia X, Wang T. Cortisol is associated with low frequency of interleukin 10-producing $\mathrm{B}$ cells in patients with atherosclerosis. Cell Biochem Funct. (2017) 35:178-183. doi: 10.1002/ cbf.3262

10. Moore KW, de Waal Malefyt R, Coffman RL, O'Garra A. Interleukin10 and the interleukin-10 receptor. Annu Rev Immunol. (2001) 19:683765. doi: 10.1146/annurev.immunol.19.1.683

11. Windsor WT, Syto R, Tsarbopoulos A, Zhang R, Durkin J, Baldwin $\mathrm{S}$, et al. Disulfide bond assignments and secondary structure analysis of human and murine interleukin 10. Biochemistry. (1993) 32:880715. doi: 10.1021/bi00085a011

12. Vieira P, de Waal-Malefyt R, Dang MN, Johnson KE, Kastelein R, Fiorentino DF, et al. Isolation and expression of human cytokine synthesis inhibitory factor cDNA clones: homology to Epstein-Barr virus open reading frame BCRFI. Proc Natl Acad Sci USA. (1991) 88:11726. doi: $10.1073 /$ pnas.88.4.1172

13. Moore KW, O'Garra AR, de Waal M, Vieira P, Mosmann TR. Interleukin-10. Annu Rev Immunol. (1993) 11:16590. doi: 10.1146/annurev.iy.11.040193.001121

14. Rashed R, Shafik RE, Shafik NF, Shafik HE. Associations of interleukin10 gene polymorphisms with acute myeloid leukemia in human (Egypt). J Cancer Res Ther. (2018) 14:1083-6. doi: 10.4103/0973-1482.1 87367

15. Xue H, Lin B, An J, Zhu Y, Huang G. Interleukin-10-819 promoter polymorphism in association with gastric cancer risk. BMC Cancer. (2012) 12:102. doi: 10.1186/1471-2407-12-102

16. Zhou Y, Li N, Zhuang W, Liu G, J, Wu T, Wu X, et al. Interleukin10-1082 promoter polymorphism associated with gastric cancer among Asians. Eur J Cancer. (2008) 44:2648-54. doi: 10.1016/j.ejca.2008. 07.017

17. Hofmann SR, Moller J, Rauen T, Paul D, Gahr Z, Hedrich M, et al. dynamic CpG-DNA methylation of Il10 and Il19 in CD4+ T lymphocytes and macrophages: effects on tissue-specific gene expression. Klin Padiatr. (2012) 224:53-60. doi: 10.1055/s-0031-1291359

18. Larsson L, Thorbert-Mros S, Rymo L, Berglundh T. Influence of epigenetic modifications of the interleukin-10 promoter on IL10 gene expression. Eur J Oral Sci. (2012) 120:14-20. doi: 10.1111/j.1600-0722.2011. 00917.x

19. Hedrich CM, Rauen T, Apostolidis SA, Grammatikos AP, Rodriguez Rodriguez N, Tsokos C, et al. Stat3 promotes IL-10 expression in lupus $\mathrm{T}$ cells through trans-activation and chromatin remodeling. Proc Natl Acad Sci USA. (2014) 111:13457-62. doi: 10.1073/pnas.1408 023111

20. Yu HR, Tsai CC, Chang LS, Huang HH, Cheng JY, Wang J, et al. l-arginine-dependent epigenetic regulation of interleukin-10, but not transforming growth factor-beta, production by neonatal regulatory $\mathrm{T}$ lymphocytes. Front Immunol. (2017) 8:487. doi: 10.3389/fimmu.2017. 00487

21. Hwang W, Lee CG, Lee C, Verma R, Rudra D, Im SH, et al. Locus-specific reversible DNA methylation regulates transient IL-10 expression in Th1 cells. J Immunol. (2018) 200:1865-75. doi: 10.4049/jimmunol.1701162

22. Lorente-Sorolla C, Garcia-Gomez A, Catala-Moll F, Toledano V, Ciudad L, Avendano-Ortiz J, et al. Inflammatory cytokines and organ dysfunction associate with the aberrant DNA methylome of monocytes in sepsis. Genome Med. (2019) 11:66. doi: 10.1186/s13073-019-0674-2

23. Qi ZX, Yu SX, Hao HS, Li FC, Guo YC, Wang K. [The analysis of IL-10 and its methylation in the patients with acute on chronic liver failure]. Zhonghua Shi Yan He Lin Chuang Bing Du Xue Za Zhi. (2011) 25:99-101.

24. Prunicki M, Stell L, Dinakarpandian DM, de Planell-Saguer RW, Lucas SK, Hammond JR, et al. Exposure to $\mathrm{NO}_{2}, \mathrm{CO}$, and PM2. (5) is linked to regional DNA methylation differences in asthma. Clin Epigenetics. (2018) 10:2. doi: 10.1186/s13148-017-0433-4

25. Son KS, Kang HS, Kim SJ, Jung SY, Min SY, Lee SY, et al. Hypomethylation of the interleukin-10 gene in breast cancer tissues. Breast. (2010) 19:484-8. doi: 10.1016/j.breast.2010. 05.011

26. Drennan S, D'Avola A, Gao Y, Weigel C, Chrysostomou E, Steele A, et al. IL-10 production by CLL cells is enhanced in the anergic IGHV mutated subset and associates with reduced DNA methylation of the IL10 locus. Leukemia. (2017) 31:1686-94. doi: 10.1038/leu.2016.356

27. Viana MB, Cardoso FP, Diniz MG, Costa FO, da Costa JE, Moreira PR, et al. Methylation pattern of IFN-gamma and IL-10 genes in periodontal tissues. Immunobiology. (2011) 216:936-41. doi: 10.1016/j.imbio.2011. 01.006

28. Fu LH, Cong B, Zhen YF, Li S, Ma CL, Ni ZY, et al. [Methylation status of the IL-10 gene promoter in the peripheral blood mononuclear cells of rheumatoid arthritis patients]. Yi Chuan. (2007). 29:1357-61. doi: 10.1360/yc-00 7-1357

29. Lin SY, Hsieh SC, Lin YC, Lee CN, Tsai MH, Lai LC, et al. A whole genome methylation analysis of systemic lupus erythematosus: hypomethylation of the IL10 and IL1R2 promoters is associated with disease activity. Genes Immun. (2012) 13:214-20. doi: 10.1038/gene.2011.74

30. Zhao M, Tang J, Gao F, Wu X, Liang Y, Yin HQ, et al. Hypomethylation of IL10 and IL13 promoters in CD4+ T cells of patients with systemic lupus erythematosus. J Biomed Biotechnol. (2010) 2010:931018. doi: 10.1155/2010/931018

31. Zhu L, Jia L, Liu Z, Zhang Y, Wang J, Yuan ZR, et al. Elevated methylation of FOXP3 (Forkhead Box P3)-TSDR (regulatory Tcell-specific demethylated region) is associated with increased risk for adverse outcomes in patients with acute coronary syndrome. Hypertension. (2019) 74:581-9. doi: 10.1161/HYPERTENSIONAHA.119. 12852

32. Shen $\mathrm{X}, \mathrm{He} \mathrm{Z}$, Li H, Yao C, Zhang Y, He L, et al. Distinct functional patterns of gene promoter hypomethylation and hypermethylation in cancer genomes. PLoS ONE. (2012) 7:e44822. doi: 10.1371/journal.pone.00 44822

33. Lima SC, Hernandez-Vargas H, Simao T, Durand G, Kruel C, Le Calvez-Kelm F, et al. Identification of a DNA methylome signature of esophageal squamous cell carcinoma and potential epigenetic biomarkers. Epigenetics. (2011) 6:1217-27. doi: 10.4161/epi.6.10. 17199

34. Tonon S, Mion F, Dong J, Chang HD, Dalla E, Pucillo CE, et al. IL-10-producing $\mathrm{B}$ cells are characterized by a specific methylation signature. Eur J Immunol. (2019) 49:1213-25. doi: 10.1002/eji.201 84825

35. Saraggi D, Fassan M, Mescoli C, Scarpa M, Valeri N, Michielan A, et al. The molecular landscape of colitis-associated carcinogenesis. Dig Liver Dis. (2017) 49:326-30. doi: 10.1016/j.dld.2016.12.011

36. Papait R, Cattaneo P, Kunderfranco P, Greco C, Carullo P, Guffanti A, et al. Genome-wide analysis of histone marks identifying an epigenetic signature of promoters and enhancers underlying cardiac hypertrophy. Proc Natl Acad Sci USA. (2013) 110:20164-9. doi: 10.1073/pnas.13151 55110

37. Cherng $\mathrm{CH}$, Lee $\mathrm{KC}$, Chien CC, Chou CK, Cheng YC, Hsin ST, et al Baicalin ameliorates neuropathic pain by suppressing HDAC1 expression in the spinal cord of spinal nerve ligation rats. J Formos Med Assoc. (2014) 113:513-20. doi: 10.1016/j.jfma.2013.04.007

38. Denk F, McMahon SB. Chronic pain: emerging evidence for the involvement of epigenetics. Neuron. (2012) 73:435-44. doi: 10.1016/j.neuron.2012.01.012

39. Bai AH, Wu WK, Xu L, Wong S, Go MY, Chan AW, et al. Dysregulated lysine acetyltransferase $2 \mathrm{~B}$ promotes inflammatory bowel disease pathogenesis through transcriptional repression of interleukin-10. J Crohns Colitis. (2016) 10:726-34. doi: 10.1093/ecco-jcc/jjw020

40. Mukherjee S, Mukherjee B, Mukhopadhyay R, Naskar K, Sundar S, Dujardin $\mathrm{J}$, et al. Imipramine exploits histone deacetylase 11 to increase the IL-12/IL-10 ratio in macrophages infected with antimony-resistant Leishmania donovani and clears organ parasites in experimental infection. J Immunol. (2014) 193:4083-94. doi: 10.4049/jimmunol.1400710

41. Duan L, Yi M, Chen J, Li S, Chen W. Mycobacterium tuberculosis EIS gene inhibits macrophage autophagy through up-regulation of IL-10 by increasing the acetylation of histone H3. Biochem Biophys Res Commun. (2016) 473:1229-34. doi: 10.1016/j.bbrc.2016.04.045 
42. Kim VN, Han J, Siomi MC. Biogenesis of small RNAs in animals. Nat Rev Mol Cell Biol. (2009) 10:126-39. doi: 10.1038/nrm2632

43. Takuse Y, Watanabe M, Inoue N, Ozaki R, Ohtsu H, Saeki M, et al. Association of IL-10-regulating microRNAs in peripheral blood mononuclear cells with the pathogenesis of autoimmune thyroid disease. Immunol Invest. (2017) 46:590-602. doi: 10.1080/08820139.2017.1322975

44. Khorrami S, Zavaran Hosseini A, Mowla SJ, Soleimani M, Rakhshani N, Malekzadeh R. MicroRNA-146a induces immune suppression and drug-resistant colorectal cancer cells. Tumour Biol. (2017) 39:1010428317698365. doi: 10.1177/10104283176 98365

45. Zhang Q, Xin H, Fen T. Function of microRNA141 in human breast cancer through cytotoxic CD4+ $\mathrm{T}$ cells regulated by MAP4K4 expression. Mol Med Rep. (2018) 17:7893-01. doi: 10.3892/mmr.201 8.8814

46. Gan L, Sun T, Li B, Tian J, Zhang J, Chen X, et al. Serum miR-146a and miR-150 as potential new biomarkers for hip fracture-induced acute lung injury. Mediators Inflamm. (2018) 2018:8101359. doi: 10.1155/2018/81 01359

47. Moradi N, Fadaei R, Ahmadi R, Kazemian E, Fallah S. Lower expression of miR-10a in coronary artery disease and its association with pro/anti-inflammatory cytokines. Clin Lab. (2018) 64:847-54. doi: 10.7754/Clin.Lab.2018. 171222

48. Lochhead RB, Ma Y, Zachary JF, Baltimore D, Zhao JL, Weis JH, et al. MicroRNA-146a provides feedback regulation of lyme arthritis but not carditis during infection with Borrelia burgdorferi. PLoS Pathog. (2014) 10:e1004212. doi: 10.1371/journal.ppat.1004212

49. Kurowska-Stolarska M, Alivernini S, Ballantine LE, Asquith DL, Millar NL, Gilchrist DS, et al. MicroRNA-155 as a proinflammatory regulator in clinical and experimental arthritis. Proc Natl Acad Sci USA. (2011) 108:111938. doi: 10.1073/pnas.1019536108

50. Fairfax KA, Gantier MP, Mackay F, Williams B, McCoy CE. IL-10 regulates Aicda expression through miR-155. J Leukoc Biol. (2015). 97:718. doi: 10.1189/jlb.2A0314-178R

51. Lochhead RB, Zachary JF, Dalla Rosa L, Ma Y, Weis JH, O'Connell RM, et al. Antagonistic interplay between MicroRNA-155 and IL-10 during lyme carditis and arthritis. PLoS ONE. (2015) 10:e0135142. doi: 10.1371/journal.pone.0135142

52. Ren Z, Q, Liu N, Zhao K. Micro RNA-19a suppresses IL-10 in peripheral B cells from patients with atherosclerosis. Cytokine. (2016) 86:8691. doi: 10.1016/j.cyto.2016.07.019

53. Geng XR, Qiu SQ, Yang LT, Liu ZQ, Yang G, Liu JQ, et al. Allergenspecific immune response suppresses interleukin 10 expression in B cells via increasing micro-RNA-17-92 cluster. Cell Biochem Funct. (2016). 34:44954. doi: 10.1002/cbf.3207

54. Liu ZQ, Yang G, Geng XR, Liu J, Mo LH, Liu ZG, et al. Micro RNA-17-92 cluster mediates interleukin-4-suppressed IL-10 expression in B cells. Am J Transl Res. (2016) 8:2317-24.

55. Chen J, Zhao L, Wang D, Xu Y, Gao H, Tan W, et al. Contribution of regulatory $\mathrm{T}$ cells to immune tolerance and association of microRNA210 and Foxp3 in preeclampsia. Mol Med Rep. (2019) 19:1150-8. doi: 10.3892/mmr.2018.9733

56. Luo Y, Huang Y, Y, Jin Y, Li CR, Yang J. [Effect of miR-21 on the expression of interleukin-10 in B cell of patients with Henoch-Schonlein purpura]. Zhonghua $\mathrm{Er} \mathrm{Ke} \mathrm{Za}$ Zhi. (2018) 56:939-44. doi: 10.3760/cma.j.issn.0578-1310.2018.12.010

57. Wang S, Li Z, Chen Q, Wang L, Zheng J, Lin Z, et al. NF-kappaBInduced MicroRNA-211 inhibits interleukin-10 in macrophages of rats with lipopolysaccharide-induced acute respiratory distress syndrome. Cell Physiol Biochem. (2018) 45:332-42. doi: 10.1159/0004 86814

58. Lu MC, Yu CL, Chen HC, Huang HB, Lai NS. Increased miR223 expression in $\mathrm{T}$ cells from patients with rheumatoid arthritis leads to decreased insulin-like growth factor-1-mediated interleukin-10 production. Clin Exp Immunol. (2014) 177:641-51. doi: 10.1111/cei. 12374

59. Sun Y, Guo D, Liu B, Yin X, Wei H, Tang K, et al. Regulatory role of rnomiR-30b-5p in IL-10 and toll-like receptor 4 expressions of T lymphocytes in experimental autoimmune uveitis in vitro. Mediators Inflamm. (2018) 2018:2574067. doi: 10.1155/2018/2574067

60. Liu D, Zhang N, Zhang X, Qin M, Dong Y, Jin L. MiR-410 down-regulates the expression of interleukin-10 by targeting STAT3 in the pathogenesis of systemic lupus erythematosus. Cell Physiol Biochem. (2016) 39:30315. doi: $10.1159 / 000445625$

61. Li L, Sun P, Zhang C, Li Z, Zhou W. MiR-98 suppresses the effects of tumor-associated macrophages on promoting migration and invasion of hepatocellular carcinoma cells by regulating IL-10. Biochimie. (2018) 150:2330. doi: 10.1016/j.biochi.2018.04.016

62. Li Y, Rong J, Qin J, He JY, Chen H, Huang SH. Micro RNA-98 interferes with expression interleukin-10 in peripheral B cells of patients with lung cancer. Sci Rep. (2016) 6:32754. doi: 10.1038/srep32754

63. Li J, Chen $\mathrm{C}, \mathrm{Bi} \mathrm{X}$, Zhou C, Huang $\mathrm{T}$, Ni C, et al. DNA methylation of CMTM3, SSTR2, and MDFI genes in colorectal cancer. Gene. (2017) 630:17. doi: 10.1016/j.gene.2017.07.082

64. Luo XQ, Yang SB, Qiu SQ, Xie RD, Yang LT, Ke YX, et al. Post-transcriptional regulation of interleukin-10 in peripheral $\mathrm{B}$ cells of airway allergy patients. $\mathrm{Am}$ J Transl Res. (2016) 8:5766-72.

65. Rouas R, Merimi M, Najar M, El Zein N, Fayyad-Kazan M, Berehab M, et al. Human CD8(+) CD25 (+) CD127 (low) regulatory T cells: microRNA signature and impact on TGF-beta and IL-10 expression. J Cell Physiol. (2019) 234:17459-72. doi: 10.1002/jcp.28367

66. Wang $\mathrm{H}, \mathrm{Xu}$ W, Shao Q, Ding Q. miR-21 silencing ameliorates experimental autoimmune encephalomyelitis by promoting the differentiation of IL-10-producing B cells. Oncotarget. (2017) 8:94069-79. doi: 10.18632/oncotarget.21578

67. Xiong Y, Yan C, Chen L, Endo Y, Sun Y, Zhou W, et al. IL-10 induces MC3T3E1 cells differentiation towards osteoblastic fate in murine model. J Cell Mol Med. (2020) 24:1076-1086. doi: 10.1111/jcmm.14832

68. Li L, Feng J, Zhang D, Yong J, Wang Y, Yao J, et al. Differential expression of miR-4492 and IL-10 is involved in chronic rhinosinusitis with nasal polyps. Exp Ther Med. (2019) 18:3968-76. doi: 10.3892/etm.2019.8022

69. Hatab HM, Abdel Hamid FF, Soliman AF, Al-Shafie TA, Ismail YM, El-Houseini ME. A combined treatment of curcumin, piperine, and taurine alters the circulating levels of IL-10 and miR-21 in hepatocellular carcinoma patients: a pilot study. J Gastrointest Oncol. (2019) 10:76676. doi: 10.21037/jgo.2019.03.07

70. Hussain T, Zhao D, Shah SZA, Wang J, Yue R, Liao Y, et al. MicroRNA $27 \mathrm{a}-3 \mathrm{p}$ regulates antimicrobial responses of murine macrophages infected by Mycobacterium avium subspecies paratuberculosis by targeting interleukin10 and TGF-beta-activated protein kinase 1 binding protein 2. Front Immunol. (2017) 8:1915. doi: 10.3389/fimmu.2017.01915

71. Chen X, Dong S, Zhang N, Chen L, Li MG, Song J, et al. MicroRNA98 plays a critical role in experimental myocarditis. Int J Cardiol. (2017). 229:75-81. doi: 10.1016/j.ijcard.2016.11.263

72. Guo X, Gao L, Wang Y, Chiu DK, Wang T, Deng Y. Advances in long noncoding RNAs: identification, structure prediction and function annotation. Brief Funct Genomics. (2016) 15:38-46. doi: 10.1093/bfgp/elv022

73. Tang XD, Zhang DD, Jia L, Ji W, Zhao YS. IncRNA AFAP1-AS1 promotes migration and invasion of non-small cell lung cancer via up-regulating IRF7 and the RIG-I-like receptor signaling pathway. Cell Physiol Biochem. (2018) 50:179-95. doi: 10.1159/000493967

74. Pei $\mathrm{X}$, Wang $\mathrm{X}, \mathrm{Li} \mathrm{H}$. LncRNA SNHG1 regulates the differentiation of treg cells and affects the immune escape of breast cancer via regulating miR-448/IDO. Int J Biol Macromol. (2018) 118(Pt A):2430. doi: 10.1016/j.ijbiomac.2018.06.033

75. Ye Y, Xu Y, Lai Y, He W, Li Y, Wang R, et al. Long non-coding RNA cox2 prevents immune evasion and metastasis of hepatocellular carcinoma by altering M1/M2 macrophage polarization. J Cell Biochem. (2018) 119:295163. doi: $10.1002 /$ jcb. 26509

76. Zhou WY, Zhang MM, Liu C, Kang Y, Wang JO, Yang XH. Long noncoding RNA LINC00473 drives the progression of pancreatic cancer via upregulating programmed death-ligand 1 by sponging microRNA-195-5p. J Cell Physiol. (2019) 234:23176-89. doi: 10.1002/jcp.28884

77. Ma P, Li Y, Zhang W, Fang F, Sun J, Liu M, et al. Long non-coding RNA MALAT1 inhibits neuron apoptosis and neuroinflammation while stimulates neurite outgrowth and its correlation with MiR-125b mediates PTGS2, CDK5 
and FOXQ1 in Alzheimer's disease. Curr Alzheimer Res. (2019) 16:596612. doi: 10.2174/1567205016666190725130134

78. Shi Y, L, Wang Q, Wei JC. Influence of IncRNA-MALAT1 on neuronal apoptosis in rats with cerebral infarction through regulating the ERK/MAPK signaling pathway. Eur Rev Med Pharmacol Sci. (2019) 23:8039-48. doi: 10.26355/eurrev_201909_19020

79. Li Y, Li Y, Huang S, He K, Zhao M, Lin H, et al. Long non-coding RNA growth arrest specific transcript 5 acts as a tumour suppressor in colorectal cancer by inhibiting interleukin-10 and vascular endothelial growth factor expression. Oncotarget. (2017) 8:13690-702. doi: 10.18632/oncotarget. 14625
Conflict of Interest: The authors declare that the research was conducted in the absence of any commercial or financial relationships that could be construed as a potential conflict of interest.

Copyright (๑ 2020 Zheng, Huang, Gao, Huang, Zou, Zou and Duan. This is an open-access article distributed under the terms of the Creative Commons Attribution License (CC BY). The use, distribution or reproduction in other forums is permitted, provided the original author(s) and the copyright owner(s) are credited and that the original publication in this journal is cited, in accordance with accepted academic practice. No use, distribution or reproduction is permitted which does not comply with these terms. 